\title{
Assessment of Cardiopulmonary Resuscitation Expertise among Dental House Officers working in a dental hospital, Multan
}

\author{
JAVERIA AFZAL ${ }^{1}$, SAIMA RAFI ${ }^{2}$, DANISH JAVED ${ }^{3}$, SANA ZAFAR ${ }^{4}$, MAHWISH SAJID $^{5}$, RABIA MAHMOOD $^{6}$ \\ ${ }^{1}$ Senior Lecturer, Multan Medical and Dental College Multan \\ ${ }^{2}$ Demonstrator, Community Medicine, Shifa College of Medicine, Islamabad \\ ${ }^{3}$ Associate Professor, Department of Science of Oral Pathology, Islam Dental College Sialkot \\ ${ }^{4}$ Associate Professor, Department of Science of Oral Biology, Islam Dental College Sialkot \\ ${ }^{5}$ WMO, Punjab Institute of Cardiology, Lahore \\ ${ }^{6}$ Assistant professor, Community Medicine, Federal Medical College, SZABMU Islamabad \\ Correspondence to Dr. Javeria Afzal
}

\begin{abstract}
Background: Cardiopulmonary arrest (CPA) is an abrupt and an unpredicted halt in patients' breathing as well as circulation due to several reasons. All wellbeing experts, including dental specialists, should be very much prepared to take care of and oversee health related crises.

Aim: To examine House Surgeon's medical practices of the recent CPR guidelines and to recognize the precautions that should be followed to correct the deficiencies identified.

Setting: Multan Dental College Multan

Methodology: Participants were selected randomly. Knowledge about CPR was evaluated by printed objective paper. Practical expertise/ skills were evaluated by SimMan (high-fidelity simulator). Objective paper comprised of 7 multiple choice questions and eighteen true false. Allotted time duration was 20 Minutes. One mark for every query and $50 \%$ marks were thought-off as passing scores

Results: $34 \%$ of the participants scored $50 \%$ or more marks while $66 \%$ failed to do so. Regarding practical demonstration's none of the participant was successful completely. Failure in initial assessment was attributed to $67 \%$ participants. Failure due to compression rate error, Failure due to ventilation rate error \& Failure due to wrong hand position were reported by $70 \%$ participants.

Conclusion: We conclude that level of knowledge as well as training regarding medicinal emergencies of dental house officers is below the required standard. Therefore, it is essential to place correct strategies \& plans in place to fortify their recognized zones of weakness.
\end{abstract}

Keywords: Cardiopulmonary Resuscitation (CPR), Dental House officers, Cardiopulmonary arrest (CPA),

\section{INTRODUCTION}

Cardiac arrest considered as a main source of demise in many parts of the globe. Cardiopulmonary arrest (CPA) is an abrupt and an unpredicted halt in patients' breathing as well as circulation due to several reasons ${ }^{1-7}$. All wellbeing experts, including dental specialists, should be very much prepared to take care of and oversee health related crises. Other than preparing in fundamental methods of resuscitation, for instance mouth to mouth ventilation joined with heart pressure, different techniques can likewise be helpful. Dental specialists ought to have nearby and be prepared to utilize an oropharyngeal tube, a laryngoscope, an oxygen balloon, an Ambu mask as well as drugs, for example lidocaine, epinephrine ${ }^{8}$.

Cardiopulmonary resuscitation (CPR) can twofold or significantly increase the probability of survival afterwards cardiovascular arrest ${ }^{9}$. Cardiopulmonary resuscitation (CPR) incorporates all the hard work and practices for reviving an individual who is in heart failure. For as long as 50 years or thereabouts, early acknowledgment, defibrillation, actuation, quick CPR and the fundamental standards of getting urgent clinical care or consideration have saved a huge number of individuals' lives around the

Received on 11-05-2021

Accepted on 21-09-2021 world. These examples illustrate the significance of resuscitation investigates and utilization of those practices in various clinics ${ }^{10}$

In the recent rules, CPR is inspected under two subheadings including advanced cardiac life support (ACLS) and essential or basic life support (BLS), which are ensuing and indivisible. During oral treatment, it was stated that instances of heart arrest were noticed on certain events, despite the fact that they were uncommon. The wellbeing experts including dental specialists should be decidedly ready for medically urgent conditions ${ }^{7}$

In dental centers, there is a chance of encountering health related crises or medical emergencies (ME). However, the quantity of health-related crises cases has prominently amplified with the expanding number of old patients having clinical problems. The cases revealed have commonly been syncope, hypertensive emergency etc. In an investigation led in 2000s, 20 instances of death were accounted for more than 10 years. Amongst the ME cases experienced in dental facilities, the CPA's cases rate was $1.1 \%-1.4 \%^{7}$

Like all other healthcare professionals, Cardiopulmonary resuscitation expertise or skills are also compulsory for each dentist. But regrettably, dental surgeons are almost deprived off from the clinical skills and knowledge regarding cardiopulmonary resuscitation ${ }^{11}$.

The purpose of this investigation was to examine House Surgeon's medical practices of the recent CPR 
guidelines and to recognize the precautions that should be followed to correct the deficiencies identified.

\section{METHODOLOGY}

CPR is defined by American Heart Association as in case of cardiac arrest to the patient with neither pulse nor breathing, a rescuer should commence with 30 rib cage compressions followed by 2 breaths ${ }^{10}$. House surgeons from Multan Dental College, Multan after permission from IRB, were the participants which were selected randomly. Knowledge about CPR was evaluated by printed objective paper. Practical expertise/ skills were evaluated by SimMan (high-fidelity simulator). Objective paper comprised of 7 multiple choice questions and eighteen true false. Allotted time duration was 20 Minutes. One mark for every query and fifty percent marks were thought-off as passing scores. Topics tested are shown in Table 1.

The SimMan (a high-fidelity simulator) applied test was used to check the capability of candidate to accomplish CPR. Superior consideration was paid to preliminary assessment, rate and volume, ventilation, force, rhythm \& rate of compression. As stated by American heart association 2015 guiding principle following criteria of CPR was evaluated;

Checkered for responsiveness is only gasping or no breathing (i.e., no normal breathing) as well as no certain pulse recorded within ten seconds (Pulse check \& Breathing can be done concurrently in $<10$ seconds)

Both hands must be on the lesser half of sternum Compression amount is altered to a range of one hundred to $120 / \mathrm{min}$.

Compression penetration for grownups is altered to minimum 2 inches $(5 \mathrm{~cm})$ but then again should not surpass $6 \mathrm{~cm}$ (2.4 inches).

To permit complete chest wall recoil afterwards every compression, saviors should evade leaning on the chest amongst compressions.

The savior is to initiate trunk compressions beforehand giving rescue breaths (C-A-B instead of $A-B-C$ ) to lessen delay to $1^{\text {st }}$ compression. The solo rescuer must begin CPR with thirty chest compressions immediately followed by 2 breaths.

For those individuals with an advanced airline in place and continuing CPR, a simplified rate of ventilation of one breath each 6 seconds (ten breaths each minute) is suggested.

\section{RESULTS}

Form was accomplished by fifty House officers. Males were twenty while females were Thirty (Table 1). Result of Objective Paper has been tabulated in Table 2. Test Pattern (MCQ's) has been demonstrated in Table 3. Graph 1 shows the results of performance assessment of CPR

Table 1: Gender distribution

\begin{tabular}{|l|c|c|}
\hline Male & Female & Total \\
\hline $20(40 \%)$ & $30(60 \%)$ & 50 \\
\hline
\end{tabular}

Table 2: Result of objective paper

\begin{tabular}{|c|c|c|}
\hline Pass (Achieving $\mathbf{5 0} \%$ or more marks & Fail (Less than $\mathbf{5 0} \%$ marks) & $\mathbf{n}$ \\
\hline $17(34 \%)$ & $33(66 \%)$ & 50 \\
\hline
\end{tabular}

Table 3: test pattern (MCQs)

\begin{tabular}{|l|c|}
\hline Topic & Number of questions \\
\hline General theory about CPR & 10 \\
\hline Ventilation & 5 \\
\hline Managing Ventricular fibrillation & 3 \\
\hline Management of Asystole & 3 \\
\hline Ventricular extrasystole & 2 \\
\hline Drugs/Dosage & 1 \\
\hline Electromechanical dissociation & 1 \\
\hline
\end{tabular}

Graph 1: Results of performance assessment of cpr

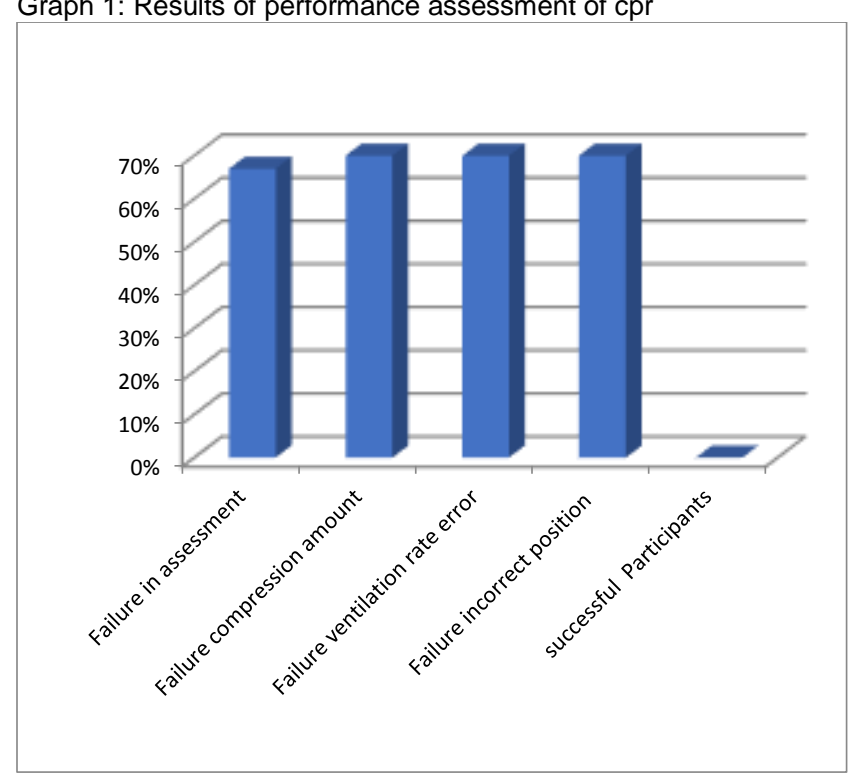

\section{DISCUSSION}

Several medicinal emergency cases may be reported in dental setup $^{12}$. Those may consist of an extensive range of circumstances from syncope, hypertensive crisis, and angina pectoris to CPA cases. Dental surgeons are also categorized as healthcare professionals; thus, it is also obligatory for them to have complete knowledge \& skills to treat with $\mathrm{ME}^{11}$.

The results of the study conducted by Mustafa Sajid clearly showed that the participants did not have the requisite skills and trainings to deal with a medicinal emergency which is similar to this study ${ }^{11}$. In this study, $34 \%$ participants were pass in the objective paper which is almost similar to the findings of another study $(41 \%)^{13}$

Satisfactory knowledge as well as appropriate awareness of practices \& techniques permit a person to successfully resuscitate and save the life of the victim. Every healthcare worker should always be aware $\&$ trained practically about BLS ${ }^{14-16}$.

In our study, we looked for retaining of basic life care expertise in dental house surgeons, undergoing the standard training and preparation protocol where almost all students failed the exam as in the study of Pim A. de Ruijter ${ }^{17,18}$. Expertise to effectively checkered vital signs as well as initiate CPR when suitable were conserved longer ${ }^{19}$. Several other studies also showed that hands-on expertise in resuscitation lessen rapidly ${ }^{20}$. In this examination, majority subjects effectively evaluate vital signs, but they were unsuccessful to preserve adequate depth of chest compression as well as ventilation volumes; we consider this delay in hands-on expertise may be due to lack of chances for hands-on practice ${ }^{18}$. Many other researches have scrutinized the awareness regarding BLS knowledge 
among healthcare workers \& different other communities ${ }^{21,22,23}$.

In this study, $70 \%$ participants faced a failure due to compression \& ventilation rate errors which is far much greater than the findings reported by another researcher where just $1.2 \%$ of the subjects were fully conscious about the general compression-ventilation ratio \& $20.4 \%$ were conscious of the order of $\mathrm{CPR}^{24}$. In another study by Zeinab Mohammed, $26.7 \%$ of junior doctor's aware of/succeeded in Rate of chest compressions which is in accordance to our study here $30 \%$ succeeded while $70 \%$ failed 25 .

Participants failed in certain critical features of CPR. Furthermore, not a major number of subjects accomplish precise CPR on the simulator. Incompetently following to AHA guiding principle demonstrates numerous key factors. Several other studies display that BLS practice may be hard to recall under tension or stress, as noticed by poor CPR performance in this study. Secondly, it could be problematic for inexpert practitioners, for instance dental house surgeons, to convert appropriate protocol into real clinical performance 26 .

Results of this study demonstrates that here is a training deficiency in basic life support program. There is a requisite of regular hands-on courses and programs of basic life support which should occur on annual or biannual intervals. Colon A Graham fruitfully revealed a beneficial training consequence in the dental participants. He identified those subjects who 'qualified or passed' afterwards training, i.e. those showed capability of providing successful \& effective BLS $(71 / 75,94.7 \%)$ as well as those who were not categorized as competent or capable $(4 / 71,5.3 \%)^{27}$. Thus, consistent preparation sessions for BLS must be compulsory \& usage of several media devices are also recommended for dental health care workers to recall the knowledge, awareness as well as clinical abilities ${ }^{28}$

\section{CONCLUSION}

We conclude that level of knowledge as well as training regarding medicinal emergencies of dental house officers is below the required standard. Therefore, it is essential to place correct strategies \& plans in place to fortify their recognized zones of weakness.

\section{Conflict of interest: Nil}

\section{REFERENCES}

1. Nishiyama C Long-term Retention of Cardiopulmonary Resuscitation Skills After Shortened Chest Compression-only Training and Conventional Training: A Randomized Controlled Trial. Acad emerg medicine January 2014; 21(1):47-54.

2. Yow AG, Rajasurya V, Sharma S. Sudden cardiac death. StatPearls [Internet]. 2020 May 30

3. Wong CX, Brown A, Lau DH, Chugh SS, Albert CM, Kalman JM, Sanders P. Epidemiology of sudden cardiac death: global and regional perspectives. Heart, Lung and Circulation. 2019 Jan 1;28(1):6-14.

4. American Heart Association. 2010 American Heart Association guidelines for cardiopulmonary resuscitation and emergency cardiovascular care. Circulation. 2010;112(Supp 3): S640-946. 2.

5. https://my.clevelandclinic.org/health/diseases/17522-sudden-cardiacdeath-sudden-cardiac-arrest 2019 May 14
6. Grant Fletcher, Thomas Rea. Quah SR. International encyclopedia of public health. Academic Press; 2017 Second Edition

7. Yükse IM,EryiğitV,Karaaslan U,SağlamC.Dentists'Attitudes Toward Up-To-Date Cardiopulmonary Resuscitation Guidelines. Eurasian J Emerg Med 2015; 14: 177-82.

8. Kavari $\mathrm{SH}$, Chohedri $\mathrm{AH}$. Cardiopulmonary Resuscitation: Knowledge and Personal Experience In Iranian Dentists. Pak J Med Sci April 2007;23(2):296-97.

9. https://www.heart.org/en/news/2018/07/12/cpr-is-key-to-survival-ofsudden-cardiac-arrest

10. Travers AH, Rea TD, Bobrow BJ, Edelson DP, Berg RA, Sayre MR, et al. Part 4: CPR overview: 2010 American Heart Association Guidelines for Cardiopulmonary Resuscitation and Emergency Cardiovascular Care. Circulation 2010;122(Suppl 3): S676-84.

11. Sajid M, Jamil. M, Kouser R, Naz S \& Javeed M. Evaluation of Cardiopulmonary Resuscitation skills of dental students \& House Officers. Pakistan Oral \& Dental Journal. 2017 Mar 31;37 (1).

12. Awata N, Hitosugi T, Miki Y, Tsukamoto M, Kawakubo Y, Yokoyama T. Usefulness of a stool to stabilize dental chairs for cardiopulmonary resuscitation (CPR). BMC emergency medicine. 2019 Dec;19(1):1-7.

13. Owaid Alsharari A, Alduraywish A, Ali Al-Zarea E, Ibrahim Salmon N, Ali Sheikh MS. Current status of knowledge about cardiopulmonary resuscitation among the university students in the northern region of Saudi Arabia. Cardiology research and practice. 2018 Jun 10;2018.

14. Almesned A, Almeman A, Alakhtar AM, et al. Basic life support knowledge of healthcare students and professionals in the Qassim University. Int J Health Sci (Qassim). 2014;8: 141-150.

15. Al Enizi BA, Saquib N, Zaghloul MS, Alaboud MS, Shahid MS, Saquib J. Knowledge and attitudes about basic life support among secondary school teachers in Al-Qassim, Saudi Arabia. Int J Health Sci (Qassim). 2016; 10:415-422.

16. Ahmad A, Akhter N, Mandal RK, Areeshi MY, Lohani M, Irshad M Alwadaani M, Haque S. Knowledge of basic life support among the students of Jazan University, Saudi Arabia: Is it adequate to save a life? Alexandria journal of medicine. 2018;54(4):555-9.

17. American Heart Association. Highlights of the 2015 American Heart Association; Guidelines update for CPR and ECC:1-32. www.heart.org/cpr.

18. Ruijter PAD et al.Retention of first aid and basic life support skills in undergraduate medical students. Med Educ Online 2014; 19:1-10.

19. Woollard M, Whitfeild R, Smith A, Colquhoun M, Newcombe RG, Vetteer $\mathrm{N}$, et al. Skill acquisition and retention in automated external defibrillator (AED) use and CPR by lay responders: a prospective study. Resuscitation 2004; 60: 17-28.

20. Madden C. Undergraduate nursing students' acquisition and retention of CPR knowledge and skills. Nurse Educ Today 2006; 26: 218-27.

21. Al-Mohaissen MA. Knowledge and attitudes towards basic life support among health students at a Saudi Women's University. Sultan Qaboos Univ Med J.2017; 17 (1):59-65

22. Alotaibi O, Alamri F, Almufleh L, Alsougi W. Basic life support: knowledge and attitude among dental students and Staff in the College of Dentistry, King Saud University. The Saudi J Dent Res. 2016; 7:5156.

23. Srinivas HT, Kotekar N, Rao RS. A survey of basic life support awareness among final year undergraduate medical, dental, and nursing students. Int J Health Allied Sci. 2014; 3:91-94.

24. Kumari KM, Amberkar MB, Alur SS, Bhat PM, Bansal S. Clinical Awareness of Do's and Don'ts of Cardiopulmonary Resuscitation (CPR) Among University Medical Students-A Questionnaire Study. Journal of clinical and diagnostic research: JCDR. 2014 Jul 20;8(7):MC08-11.

25. Mohammed Z, Arafa A, Saleh Y, Dardir M, Taha A, Shaban H, AbdelSalam EM, Hirshon JM. Knowledge of and attitudes towards cardiopulmonary resuscitation among junior doctors and medical students in Upper Egypt: cross-sectional study. International journal of emergency medicine. 2020 Dec; 13:1-8.

26. Behrend $T$ et al. Retention of cardiopulmonary resuscitation skills in medical students utilizing a high-fidelity patient simulator. Medical Student Research Journal. 2011;01(1):1-4.

27. Graham CA, Lewis NF. A scoring system for the assessment of basic life support ability.J.Eur Resc Counl. 2000;43(2):111-14.

28. Oteir AO, Almhdawi KA, Kanaan SF, Alwidyan MT, Williams B. Cardiopulmonary resuscitation level of knowledge among allied health university students in Jordan: a cross-sectional study. BMJ open. 2019 Nov 1;9(11): e031725. 